\title{
p.Ser1235Arg should no longer be considered as a cystic fibrosis mutation: results from a large collaborative study
}

\author{
Céline René ${ }^{\star 1,2,3}$, Damien Paulet ${ }^{1,2}$, Emmanuelle Girodon ${ }^{4}$, Catherine Costa ${ }^{4}$, Guy Lalau ${ }^{5}$, Julie Leclerc ${ }^{5}$, \\ Faïza Cabet-Bey ${ }^{6}$, Thierry Bienvenu ${ }^{7}$, Martine Blayau ${ }^{8}$, Albert Iron ${ }^{9}$, Hervé Mittre ${ }^{10}$, Delphine Feldmann ${ }^{11}$, \\ Caroline Guittard $^{3}$, Mireille Claustres ${ }^{1,2,3}$ and Marie des Georges ${ }^{3}$
}

\begin{abstract}
Among the 1700 mutations reported in the cystic fibrosis transmembrane conductance regulator (CFTR) gene, a missense mutation, p.Ser1235Arg, is a relatively frequent finding. To clarify its clinical significance, we collected data from 104 subjects heterozygous for the mutation p.Ser1235Arg from the French CF network, addressed for various indications including classical CF, atypical phenotypes or carrier screening in subjects with or without a family history. Among them, 26 patients ( 5 having CF, 10 CBAVD (congenital bilateral absence of the vas deferens) and 11 with CF-like symptoms) and 14 healthy subjects were compound heterozygous for a second CFTR mutation. An exhaustive CFTR gene analysis identified a second mutation in cis of p.Ser1235Arg in all CF patients and in 81.8\% CBAVD patients. Moreover, epidemiological data from $>2100$ individuals found a higher frequency of p.Ser1235Arg in the general population than in CF or CBAVD patients. These data, added to the fact that in silico analysis and functional assays suggest a benign nature of this substitution, give several lines of evidence against an association of p.Ser1235Arg with CF or CBAVD.
\end{abstract}

European Journal of Human Genetics (2011) 19, 36-42; doi:10.1038/ejhg.2010.137; published online 18 August 2010

Keywords: cystic fibrosis; diagnosis; genetic counseling; mutation

\section{INTRODUCTION}

Cystic fibrosis (CF, MIM 219700), one of the most frequent lifeshortening recessively inherited diseases, is caused by alterations in the CFTR gene (cystic fibrosis transmembrane conductance regulator; MIM 602421) that result in loss or impairment of CFTR-mediated ion transport across epithelial cell membrane. The clinical expression and severity of CF are highly variable. Severe phenotypes are usually associated with high concentrations of sweat chloride, early onset of pancreatic insufficiency (PI), meconium ileus at birth and severe lung disease, whereas mild phenotypes are associated with lower sweat chloride concentrations, pancreatic sufficiency (PS) and variable lung disease. Mutations in the CFTR gene are also involved in atypical phenotypes $^{1}$ such as CBAVD (congenital bilateral absence of the vas deferens; MIM 277180), mild pulmonary diseases or idiopathic chronic pancreatitis (ICP).

Since the cloning of the CFTR gene, a wide spectrum of molecular abnormalities ( $>1700$ mutations, variants or polymorphisms) has already been reported to the Cystic Fibrosis Genetic Analysis Consortium (http://www.genet.sickkids.on.ca/cftr/). However, a clear statement on the pathogenicity of a mutation is difficult to obtain, in particular for missense mutations. ${ }^{2,3}$ Moreover, the existence of at least two mutations or sequence variations on the same allele, named complex alleles, complicates genetic counseling. ${ }^{4-11}$ p.Ser1235Arg (3837T $>$ G or c.3705T $>$ G), initially reported by Cuppens et al. ${ }^{12}$ with a second mutation on the same allele, p.Gly628Arg, is located in a poorly conserved region in the second nucleotide binding fold (NBF2). Earlier reports failed to establish a clear impact of p.Ser1235Arg on the phenotype severity. ${ }^{13-16}$ The aim of this study was to determine whether we should consider p.Ser1235Arg as a possible CF-associated mutation or reclassify it as a neutral polymorphism. Thus, we implemented a large collaborative study to repertory the patients or individuals bearing the p.Ser1235Arg mutation from nine French laboratories and compared their phenotype, genotype and associated CFTR haplotypes. To predict the potential effect of this sequence variation on the CFTR protein, in silico analyses using various programs and functional studies were performed. Here, we gather lines of evidence that p.Ser1235Arg should be no longer considered as a CF mutation.

\section{MATERIALS AND METHODS}

Patients and individuals

Data were collected from 104 subjects heterozygous or compound heterozygous for the p.Ser1235Arg mutation registered from the French CF network of

\footnotetext{
${ }^{1}$ Université Montpellier1, UFR de Médecine, Montpellier, France; ${ }^{2}$ INSERM U827, Laboratoire de Génétique de Maladies Rares, Montpellier, France; ${ }^{3}$ CHU Montpellier, Hôpital Arnaud de Villeneuve, Laboratoire de Génétique Moléculaire, Montpellier, France; ${ }^{4}$ Service de Biochimie-Génétique et Inserm U955 équipe 11 , Groupe Henri Mondor-Albert Chenevier, APHP, Créteil, France; ${ }^{5}$ Pôle de Biochimie et Biologie Moléculaire, Centre de Biologie Pathologie, CHU de Lille, Lille, France; ${ }^{6}$ Service d’Endocrinologie Moléculaire et Maladies rares, Centre de Biologie et Pathologie Est, CHU de Lyon, Bron, France; ${ }^{7}$ Laboratoire de Biochimie-Génétique, Hôpital Cochin, APHP, Paris, France; ${ }^{8}$ Laboratoire de Génétique Moléculaire, CHU Pontchaillou, Rennes, France; ${ }^{2}$ Laboratoire de Génétique Moléculaire, Service de Génétique Médicale, Groupe Hospitalier Pellegrin, CHU de Bordeaux, Bordeaux, France; ${ }^{10}$ Laboratoire de Biochimie B, CHU Georges Clémenceau, Caen, France; ${ }^{11}$ Laboratoire de Biochimie et Biologie Moléculaire, Hôpital d'Enfants Armand Trousseau, APHP, Paris, France

*Correspondence: Dr C René, INSERM U827, Laboratoire de Génétique Moléculaire et Chromosomique, CHU, Institut Universitaire de Recherche Clinique, 641 Avenue du Doyen Gaston Giraud, Montpellier Cedex 5, 34093, France.

Tel: +33 46741 5360; Fax: +33 46741 5365; E-mail: celine.rene@inserm.fr
}

Received 22 March 2010; revised 10 June 2010; accepted 29 June 2010; published online 18 August 2010 
molecular genetics laboratories. Among them, 67 were referred for diagnosis: classical CF (6 unrelated patients), CAVD (10 individuals with bilateral CAVD (CBAVD) and one with unilateral CAVD (CUAVD)), fetal suspicion of CF (8 fetuses with abnormal ultrasound signs of bowel anomalies) and 42 individuals presenting CF-related symptoms (according to the International Classification of Disease (ICD) in the section 'Cystic Fibrosis and Related Disorders' (Meeting report, 2002)) with normal or borderline sweat chloride values including genital (5 individuals), respiratory (23 individuals) or digestive (14 individuals) symptoms. A total of 37 healthy subjects were referred for carrier screening, including 14 individuals with a positive family history and 23 partners of CF patients or carriers. Written consents to the genetic study were obtained from the patients and/or their family and from healthy subjects.

\section{Molecular epidemiological study}

To evaluate the p.Ser1235Arg frequency in the general population, we screened for 2114 samples: 929 anonymized dried-blood spot of neonates presenting positive or negative immunoreactive trypsinogen (IRT) at day 3 obtained from the center for neonatal screening in Montpellier, South of France, and 1185 genomic DNA from CF patient's or relative's partners from the cohort of the Southern France (Laboratory of Molecular Genetics of Montpellier) or from the cohort of the Northern France (Laboratory of Molecular Genetics of Lille).

\section{Mutation nomenclature}

Gene variants at the protein level were named as recommended in the Human Genome Variation Society web page (http://www.hgvs.org/mutnomen/). For variations described at the nucleotide level, the A of the ATG translation start codon was numbered as +133 in accordance with the current CFTR gene numbering based on cDNA sequence (GenBank NM_000492.3) and on the $\mathrm{CF}$ mutation database. These variations were also given in parentheses following the approved nomenclature format (A of the ATG translation start codon as +1 ).

\section{CFTR genotype analysis}

Genomic DNA. Genomic DNA was prepared from peripherical blood leukocytes or from amniotic liquid according to standard protocols. For the patients, the CFTR gene coding and flanking regions were analyzed by PCR amplification followed by denaturing gel gradient electrophoresis (DGGE), single-strand conformation analysis (SSCA), denaturing high-performance liquid chromatography (DHPLC) or heteroduplex analysis followed by sequencing each abnormal pattern detected to characterize the variants. If p.Ser1235Arg was found alone, a screening for large CFTR rearrangements was performed using a semiquantitative fluorescent multiplex PCR assay. For the patients' relatives, a screening for the familial mutations was implemented. For the patients' partners, in primary screening, 5 to 12 exons, including exons 13 and 19 , were screened by laboratories.

To determine the haplotype associated with the p.Ser1235Arg allele, six microsatellite markers (IVS1(CA), IVS8(CA), IVS8(TG)m, IVS8(T)n, $\operatorname{IVS} 17 \mathrm{~b}(\mathrm{TA})$ and $\operatorname{IVS} 17 \mathrm{~b}(\mathrm{CA})$ ) and one biallelic marker (p.Met470Val or $1540 \mathrm{~A}>\mathrm{G}(\mathrm{c} .1408 \mathrm{~A}>\mathrm{G}))$ were investigated.

Guthrie cards. Spots of $3 \mathrm{~mm}$ diameter, punched from anonymized cards, were distributed in 96-well plates and DNA was extracted using methanol extraction. ${ }^{17}$ p.Ser1235Arg was screened using DHPLC technology and each abnormal pattern was confirmed by sequencing analysis.

\section{Computer-assisted analysis}

To predict the potential pathogenicity of p.Ser1235Arg, we used PolyPhen and SIFT softwares. The PolyPhen program (http://coot.embl.de/PolyPhen/) is based on the sequence homology and the mapping of the substitution site to known protein three-dimensional structures. Results are given as 'benign', 'possibly damaging', 'probably damaging' or 'unknown'. The SIFT program (http://sift.jcvi.org/) uses sequence homology to predict whether an amino acid substitution will affect protein function and, hence, potentially alter phenotype. Results are reported as 'deleterious or not'. Consequences on the splicing machinery were evaluated by Human Splicing Finder tool (http://www.umd.be/ $\mathrm{HSF} /$ ). This program integrates all available matrices to identify ESE, ESS, ISE and ISS motifs: ESE Finder, ${ }^{18}$ RESCUE ESE, ${ }^{19}$ silencer motifs from Sironi et al. ${ }^{20}$, ESS decamers from Wang et al. ${ }^{21}$ and new algorithms to calculate the consensus values of potential splice sites and search for Branch points. To gain some insight into the potential effect of the p.Ser1235Arg mutation from a structural point of view, we used the experimental structures of MJ0796 (in complex with ATP, pdb identifier 112t) as template for modeling as previously described by Eudes et al. ${ }^{22}$ The MJ0796 structure was visualized with MBT SimpleViewer software (San Diego, CA, USA). The alignment of sequences of the two CFTR NBDs, other ABC transporters family and MJ0796 was obtained by ClustalW program (http://align.genome.jp/) and visualized using Jalview 2.3 tools (http://www.jalview.org/).

\section{In vitro functional studies}

CFTR expression plasmids, site-directed mutagenesis, minigene constructs and cells. A plasmid expressing the human CFTR gene was constructed by placing the full-length CFTR cDNA coding sequence obtained from pTG5985 plasmid, a gift from Transgene SA (Illkirch, France), in the pcDNA3(+) expression vector. The p.Ser1235Arg mutation was inserted into the pcDNA3-CFTR vector by site-directed mutagenesis using QuickChange II site-directed mutagenesis kit (Agilent Technologies, Massy, France) according to the manufacturer's instructions.

The human CFTR genomic region encompassing exon 19 with the flanking intronic sequences was amplified from a patient heterozygous for the p.Ser1235Arg using primers: FOR 5'-AATTCTGGAGCTCGAGCAGGCCTATA CAGAGCCCATT- $3^{\prime}$ and REV 5'-CTCTTAATTTGCTAGCCATTTTCAAG ATGGGAAATCTAAAACA-3' (which introduce NheI and XhoI sites in the PCR product). After enzymatic digestion, the PCR product was subcloned into the XhoI/NheI-digested pSPL3 plasmid, kindly gifted by Dr S Tuffery-Giraud (Laboratory of Molecular Genetics, Montpellier, France). One clone containing the wild-type allele and one with the mutation were retained for expression experiments. All plasmid constructs were fully sequenced.

Beas2B, a human bronchial epithelial cell line expressing CFTR, and the monkey kidney fibroblast cells, COS-7, which does not express endogenous CFTR, were cultured as previously described. ${ }^{23}$ Cells were seeded at a density of 200000 cells per $2 \mathrm{ml}$ of medium in six-well dishes. After $24 \mathrm{~h}$, cells were transfected with Polyfect transfection reagent (Qiagen, Courtaboeuf, France) according to the manufacturer's recommendations. All experiments were repeated at least twice.

RNA extraction and RT-PCR. Total RNA was extracted using RNeasy Plus kit (Qiagen, France). Reverse transcription was performed with $1 \mu \mathrm{g}$ of total RNA as previously described. ${ }^{23}$ cDNA synthesis reaction $(1 \mu \mathrm{l})$ was used for PCR analyses using a mixture of $10 \mathrm{pmol}$ of both SD6/SA2 primers and $\beta$-actin primers, $0.5 \mathrm{U}$ of Taq DNA polymerase (Ozyme, Saint Quentin Yvelines, France), and $10 \mathrm{~mm}$ of dNTPs in a $25 \mu \mathrm{l}$ volume. Amplification was performed with an initial denaturation step at $94^{\circ} \mathrm{C}$ for $5 \mathrm{~min}, 35$ cycles of $94^{\circ} \mathrm{C}$ for $30 \mathrm{~s}$, $57^{\circ} \mathrm{C}$ for $30 \mathrm{~s}$ and $72^{\circ} \mathrm{C}$ for $90 \mathrm{~s}$, followed by a final extension step at $72{ }^{\circ} \mathrm{C}$ for 7 min. PCR products were visualized on $1.5 \%$ agarose gel. Each PCR product was purified and fully sequenced.

Whole-cell extracts and western blot. Whole-cell extracts from six-well dishes were extracted and resuspended in Laemmli buffer. Proteins were separated by SDS-PAGE in a $5 \%$ acrylamide gel, transferred in a PVDF membrane and immunoblotted with antibody against CFTR (MM13-4 from Millipore, Molsheim, France).

\section{Statistical analysis}

Statistical analyses were performed using Instat software (GraphPad Software, La Jolla, CA, USA). The $\chi^{2}$ test was used to compare differences between general population $v s$ CF patients or general population $v s$ CBAVD patients. The $P$-values of $<0.05$ were considered to indicate statistical significance.

\section{RESULTS}

\section{Genotype/phenotype description of individuals carrying p.Ser1235Arg}

Among 104 subjects from 91 unrelated families reported by the 9 French laboratories, 6 patients are affected with classical CF (Table 1a). Five of them were referred for severe disease with diagnosis before one year, positive sweat test, pancreatic insufficiency and persistent 
Table 1a Genotype and phenotype of CF, CBAVD and CF-like patients carrying the p.Ser1235Arg mutation

\begin{tabular}{|c|c|c|c|}
\hline Genotype & & Phenotype & $\begin{array}{l}\text { No. of } \\
\text { subjects }\end{array}$ \\
\hline Allele 1 & Allele 2 & & \\
\hline p.Ser1235Arg;p.Arg785X & p.Phe508del & Severe CF & 2 \\
\hline p.Ser1235Arg;p.Arg785X & $N A^{a}$ & Severe CF & 1 \\
\hline $\begin{array}{l}\text { p.Ser1235Arg; } 875+1 G>A \\
\text { (c. } 743+1 C>A)\end{array}$ & $\begin{array}{l}\text { 3629delT } \\
\text { (c.3497delT) }\end{array}$ & Severe $\mathrm{CF}$ & 1 \\
\hline p.Ser1235Arg;p.Arg785X & p.Gly542X & Severe CF & 1 \\
\hline p.Ser1235Arg;(TG)13(T)5 & p.Gly551Asp & Mild CF & 1 \\
\hline p.Ser1235Arg;(TG)13(T)5 & p.Phe508del & CBAVD & 6 \\
\hline p.Ser1235Arg;(TG)13(T)5 & p.Arg1070Trp & CBAVD & 1 \\
\hline p.Ser1235Arg;(TG)13(T)5 & $\begin{array}{l}\text { p.Arg117His; } \\
\text { (T)7 }\end{array}$ & CBAVD & 1 \\
\hline p.Ser1235Arg & p.Phe508del & CBAVD & 1 \\
\hline p.Ser1235Arg & - & CBAVD & 1 \\
\hline p.Ser1235Arg;(TG)13(T)5 & p.Phe508del & CUAVD & 1 \\
\hline
\end{tabular}

\begin{tabular}{|c|c|c|c|}
\hline & & $\begin{array}{l}\text { Suspicion } \mathrm{CF} / \mathrm{mild} \\
\text { phenotype: }\end{array}$ & \\
\hline p.Ser1235Arg & - & Genital symptoms & 5 \\
\hline p.Ser1235Arg & - & Respiratory symptoms & 16 \\
\hline p.Ser1235Arg;(TG)13(T)5 & p.Phe508del & Respiratory symptoms & 2 \\
\hline p.Ser1235Arg & $\begin{array}{l}406-6 T>C \\
(\text { c. } 274-6 T>C)\end{array}$ & Respiratory symptoms & 1 \\
\hline p.Ser1235Arg & p.Tyr1092X & Respiratory symptoms & 1 \\
\hline p.Ser1235Arg & p.Glu831X & Respiratory symptoms & 1 \\
\hline p.Ser1235Arg & p.GIn493X & Respiratory symptoms & 1 \\
\hline p.Ser1235Arg & p.lle507del & Respiratory symptoms & 1 \\
\hline p.Ser1235Arg & - & Digestive symptoms & 13 \\
\hline p.Ser1235Arg & p.Gly542X & Digestive symptoms & 1 \\
\hline p.Ser1235Arg & - & $\begin{array}{l}\text { Hyperechogenic } \\
\text { fetal bowel }\end{array}$ & 5 \\
\hline p.Ser1235Arg & $\begin{array}{l}\text { p.Arg668Cys; } \\
\text { p.Arg576Ala }\end{array}$ & $\begin{array}{l}\text { Hyperechogenic } \\
\text { fetal bowel }\end{array}$ & 1 \\
\hline p.Ser1235Arg & p.Val920Met & $\begin{array}{l}\text { Hyperechogenic } \\
\text { fetal bowel }\end{array}$ & 1 \\
\hline p.Ser1235Arg & p.Phe508del & $\begin{array}{l}\text { Hyperechogenic } \\
\text { fetal bowel }\end{array}$ & 1 \\
\hline
\end{tabular}

aNA: not available; we could only test the mother and a healthy sister (the patient was deceased and the father's DNA was not available).

respiratory infections, and one had a mild phenotype with a borderline sweat test. Complete scanning of the 27 exons and their boundaries identified a second mutation on the same allele for all of them: (1) p.Arg785X, located in exon 13, predicting a truncated protein; (2) $875+1 G>A(c .743+1 G>A)$ affecting the highly conserved splice donor site sequence; and (3) the (TG) $13(\mathrm{~T}) 5$ allele that promotes CFTR exon 9 skipping in humans. ${ }^{24-26}$

Ten patients were referred for CBAVD and one for CUAVD without renal anomalies. The (TG) $13(\mathrm{~T}) 5$ allele was found in nine subjects with p.Ser1235Arg (Table 1a). Of these, eight were compound heterozygous for p.Phe508del, and two for mutations associated with CF-related diseases, ${ }^{3,27}$ (p.Arg117His;T7) and p.Arg1070Trp (http://www.genet.sickkids.on.ca/cftr/).

Among 42 patients with monosymptomatic disease carrying p.Ser1235Arg (Table 1a), eight cases (19\%) were compound heterozygotes, seven for a severe mutation and one for the intronic variant
406-6T $>$ C (c.274-6T $>$ C). Of these, five harbored the (TG) $13(T) 5$ allele associated in cis with p.Ser1235Arg. In all, 34 individuals (81\%) were heterozygous for p.Ser1235Arg: 5 with reduced sperm quality; 16 with sinopulmonary problems such as bronchiectasis, asthma, nasal polyposis and chronic obstructive pulmonary disease; and 13 with digestive symptoms (meconium ileus at birth, pancreatitis and liver disease). One of them, referred for bronchitis and recurrent infections without bronchiectasis, had a positive sweat test $(102 \mathrm{mmol} / \mathrm{l})$.

Eight fetal samples were referred for risk calculation in the cohort of hyperechogenic fetal bowel. Among them, five carried only p.Ser1235Arg and were considered simple heterozygote because extensive scanning did not reveal a second CF mutation. ${ }^{28}$ Three cases were compound heterozygous for another mutation. In the first case, a moderate hyperechogenicity was detected at 22 weeks of pregnancy, which decreased at 31 . The fetus harbored the p.Phe508del mutation on the other allele and parents decided to continue pregnancy. The second carried the rare missense mutation p.Val920Met and depressed values of digestive enzymes were observed at 17 weeks of pregnancy. In the last case, the fetus carried the complex allele (p.Gly576Ala; p.Arg668Cys), considered as a mild mutation involved in CBAVD or disseminated bronchiectasis phenotypes in adults. ${ }^{29-31}$ Although the fetus presented hyperechogenic fetal bowel, the parents were reassured regarding the risk of classical CF. Unfortunately, no data were available on the outcome of these pregnancies.

Among 37 individuals tested for cascade carrier screening, 14 were compound heterozygous for p.Ser1235Arg and another CFTR mutation (Table $1 \mathrm{~b}$ ), all being relatives of patients or carriers. Of these, 12 harbored in trans a CF-causing mutation. No symptom of CF was notified although sweat test was not performed. Notably, cases 4 and 5 were two fertile fathers carrying a class I mutation (severe) associated in trans of p.Ser1235Arg.

Frequencies of p.Ser1235Arg in the general, CF and CBAVD French populations

In 2114 subjects of the general population, 32 p.Ser1235Arg alleles were detected, representing an allelic frequency of $0.76 \%$ (Table 2). None of them had the p.Arg785X mutation. This allele frequency was compared with those of patients collated in a French study affected with CF (8/7420 alleles) or CBAVD (7/1626 alleles), 0.11 and $0.43 \%$, respectively. ${ }^{32}$ By $\chi^{2}$ statistical analysis, the p.Ser1235Arg allelic frequency is significantly higher in the general population than in CF patients $(0.76$ vs $0.11 \%, P<0.001)$ but not statistically different from the CBAVD group ( 0.76 vs $0.43 \%, P>0.1)$. Recently, six CF patients were re-analyzed. Among them, five with severe disease, harbored the p.Arg785X mutation, whereas another presenting a mild phenotype had the complex allele (p.Ser1235Arg;T5). When the p.Ser1235Arg frequencies in CF, CBAVD and the general population were compared with the frequencies of p.Phe508del (Table 2), it appears that p.Ser1235Arg is as frequent as p.Phe508del $(P=0.91)$ in the general population (respectively, 0.76 vs $0.82 \%$ ); however, its implication in classical CF is significantly different $(0.11$ vs $67.2 \%(P<0.0001)$ and $2.86 \%(P<0.0001)$, respectively).

\section{p.Ser1235Arg haplotype backgrounds}

Haplotypic markers and familial segregation were completed in 36 cases (Table 3). The analysis of haplotypes based on four intragenic CFTR markers (IVS1(CA), IVS8(CA), M470V and IVS17b(CA)) revealed that p.Ser1235Arg is always present on the same haplotype 26-17-M-13. This haplotype associated with the polyvariant (TG)12(T)7 represents $77.8 \%$ of cases. Only the IVS17b(TA) microsatellite marker shows a large variability in this study, ranging from 7 to 44 repeats. 
Table 1b Genotype and familial history of asymptomatic individuals carrying the p.Ser1235Arg mutation

\begin{tabular}{|c|c|c|c|}
\hline \multirow[t]{2}{*}{ Case no. } & \multicolumn{2}{|c|}{ Genotype } & \multirow{2}{*}{ Familial information } \\
\hline & Allele 1 & Allele 2 & \\
\hline 1 & $\begin{array}{l}\text { p.Ser1235Arg; } \\
\text { (TG)13(T)5 }\end{array}$ & p.Phe508del & $\begin{array}{l}\text { Brother of CUAVD } \\
\text { (no parental project) }\end{array}$ \\
\hline 2 & $\begin{array}{l}\text { p.Ser1235Arg; } \\
\text { (TG)13(T)5 }\end{array}$ & p.Phe508del & Sister of CUAVD \\
\hline 3 & $\begin{array}{l}\text { p.Ser1235Arg; } \\
\text { (TG)13(T)5 }\end{array}$ & p.Arg1070Trp & Sister of CBAVD \\
\hline 4 & p.Ser1235Arg & p.Gly542X & $\begin{array}{l}\text { Father of CF [p.Phe508del]+ } \\
\text { [p.Gly542X] and healthy children } \\
\text { [p.Ser1235Arg]+[(TG)11(T)5] }\end{array}$ \\
\hline 5 & p.Ser1235Arg & p.GIn493X & $\begin{array}{l}\text { Father of CF [p.Phe508del]+ } \\
\text { [p.GIn493X] }\end{array}$ \\
\hline 6 & p.Ser1235Arg & p.Phe508del & Uncle of CF (no parental project) \\
\hline 7 & p.Ser1235Arg & p.Phe508del & $\begin{array}{l}\text { Mother of CF [p.Phe508del]+ } \\
{[1717-1 \mathrm{G}>\mathrm{A}(\mathrm{c} .1585-1 \mathrm{G}>\mathrm{A})]}\end{array}$ \\
\hline 8 & p.Ser1235Arg & $\begin{array}{l}\text { 2347delG } \\
\text { (c.2215delG) }\end{array}$ & $\begin{array}{l}\text { Mother of CF [p.Phe508del]+ } \\
\text { [2347delG (c.2215delG)] }\end{array}$ \\
\hline 9 & p.Ser1235Arg & $(\mathrm{TG}) 11(\mathrm{~T}) 5$ & $\begin{array}{l}\text { Mother of non-CF fetus with } \\
\text { hyperechogenic fetal bowel } \\
\text { [p.Phe } 508 \text { del]+[(TG)11(T)5] }\end{array}$ \\
\hline 10 & p.Ser1235Arg & p.Phe508del & Sister of CBAVD \\
\hline 11 & p.Ser1235Arg & p.Phe508del & Sister of CBAVD \\
\hline 12 & p.Ser1235Arg & p.Glu831X & Sister of CF-like patient \\
\hline 13 & p.Ser1235Arg & p.Phe508del & First-cousin of CF-like patient \\
\hline 14 & p.Ser1235Arg & p.Phe508del & $\begin{array}{l}\text { A 18-month-old child with } \\
\text { prenatal diagnostic based } \\
\text { on familial history of CF }\end{array}$ \\
\hline
\end{tabular}

Table 2 Comparison of the allelic frequencies of p.Ser1235Arg and p.Phe508del in the general population, CF and CBAVD patients

\begin{tabular}{|c|c|c|c|c|c|}
\hline & \multicolumn{3}{|c|}{ Populations } & \multicolumn{2}{|c|}{ Significance (P-values) } \\
\hline & $\begin{array}{c}\text { General } \\
\text { population, } \\
\text { this study }\end{array}$ & $\begin{array}{l}\text { CF patients } \\
\quad(n=7420)\end{array}$ & $\begin{array}{c}\text { CBAVD } \\
\text { patients }^{30} \\
(\mathrm{n}=1626)\end{array}$ & $\begin{array}{c}\text { General } \\
\text { population } \\
\text { vs CF }\end{array}$ & $\begin{array}{c}\text { General } \\
\text { population } \\
\text { vs CBAVD }\end{array}$ \\
\hline p.Phe508del & $\begin{array}{c}0.82 \% \\
(n=1950)\end{array}$ & $67.2 \%$ & $21.6 \%$ & $\mathrm{~S}(P<0.05)$ & $\mathrm{S}(P<0.05)$ \\
\hline p.Ser1235Arg & $\begin{array}{c}0.76 \% \\
(n=4228)\end{array}$ & $0.11 \%$ & $0.43 \%$ & $\mathrm{~S}(P<0.05)$ & NS $(P=0.17)$ \\
\hline $\begin{array}{l}\text { Significance } \\
\text { ( } P \text {-values) } \\
\text { p.Phe508del vs } \\
\text { p.Ser1235Arg }\end{array}$ & $\begin{array}{c}\text { NS } \\
(P=0.91)\end{array}$ & $\mathrm{S}(P<0.05)$ & $\mathrm{S}(P<0.05)$ & & \\
\hline
\end{tabular}

Abbreviations: N, number of unrelated tested chromosomes; S, significant difference; NS, no significant difference.

$P$-values significant at $<0.05$

However, three complete haplotypes differing only by one repeat in the IVS17b(TA) $(35,36$ and 37) accounted for $>63 \%$ of the cases. The p.Arg785X found in CF patients in cis of the p.Ser1235Arg occurs on a single haplotype 26-17-12-7-M-35-13. The complex allele
Table 3 CFTR haplotypes for 36 fully haplotyped p.Ser1235Arg chromosomes from CF, CBAVD patients, ruled-out CF (including CF-like symptoms and fetal echogenic bowel) and general population

\begin{tabular}{|c|c|c|c|c|c|}
\hline $\begin{array}{l}\text { IVS1(CA)-IVS8(CA)- } \\
\text { M470V-IVS17b(CA) }\end{array}$ & $\begin{array}{l}\text { IVS8(TG)m- } \\
\text { IVS8(T)n }\end{array}$ & Mutation in cis & IVS1 $7 b(T A)$ & $n$ & $\%$ \\
\hline \multirow[t]{11}{*}{ 26-17-M-13 } & $12-7$ & p.Arg785X & 35 & 4 & 11.1 \\
\hline & & $\begin{array}{l}875+1 G>A \\
(\text { c. } 743+1 C>A)\end{array}$ & 36 & 1 & 2.8 \\
\hline & & None & 33 & 1 & 2.8 \\
\hline & & & 35 & 5 & 13.8 \\
\hline & & & 36 & 9 & 25 \\
\hline & & & 37 & 4 & 11.1 \\
\hline & & & 38 & 1 & 2.8 \\
\hline & & & 40 & 1 & 2.8 \\
\hline & & & 44 & 1 & 2.8 \\
\hline & & & 7 & 1 & 2.8 \\
\hline & $13-5$ & & 33 & 8 & 22.2 \\
\hline
\end{tabular}

(p.Ser1235Arg;(TG)13(T)5) characteristic of CBAVD was also found to be carried on a unique haplotype (26-17-13-5-M-33-13).

In silico predictions and functional impact on the CFTR protein

We evaluated the degree of conservation of serine 1235 residue using alignment of the nucleotide-binding domains of the human CFTR with those of $\mathrm{ABC}$ transporters, to whom the CFTR belongs (Figure 1a). The results show that the serine 1235 is poorly conserved in the $\mathrm{ABC}$ transporter family, suggesting that amino acid change in this position is possible. Serine 1235 residue located after a $\beta$ strand is not involved in anchor or structural points. ${ }^{33}$ The analysis of its position on the 3D structure of MJ0796 (Figure 1b) previously used as template for modeling the CFTR NBDs ${ }^{22}$ points that the serine 1235 is located on the exterior surface of the NBD2. Therefore, this amino acid does not participate to the NBD1/NBD2 heterodimer formation and to the ATP binding and hydrolysis. The limited effect of this amino acid change was confirmed using SIFT and PolyPhen programs. To evaluate the functional impact of the mutation on the CFTR maturation, we performed western blot analysis on whole-cell extracts from COS-7 cells transfected with either wild-type or the p.Ser1235Arg constructs (Figure 1c). The results show the presence of the fully glycosylated CFTR protein (band C) with the p.Ser1235Arg construct, suggesting that the maturation of the p.Ser1235Arg-CFTR protein is not altered. These results suggest that p.Ser1235Arg has no functional impact on the CFTR protein.

\section{Functional impact of the p.Ser1235Arg on the splicing}

According to the Splicing Sequences Finder tools, ${ }^{34}$ p.Ser1235Arg does not seem to impact the splicing of CFTR transcript. To verify this prediction, we used a minigene-based approach. Constructs containing the wild-type and mutant CFTR exon 19 and the intron-exon boundaries were inserted in the pSPL3 vector (Figure 2a). After transfection in Beas2B cell line, RNAs were extracted and subjected to RT-PCR. As shown in Figure 2b, compared with the wild type, the presence of the p.Ser1235Arg alteration does not modify the exon 19 inclusion in the mRNA. Together, these data suggest that p.Ser1235Arg has no effect on the CFTR mRNA splicing.

\section{DISCUSSION}

Since its initial description in 1993, the clinical significance of the p.Ser1235Arg mutation remains unclear. This ignorance makes genetic 

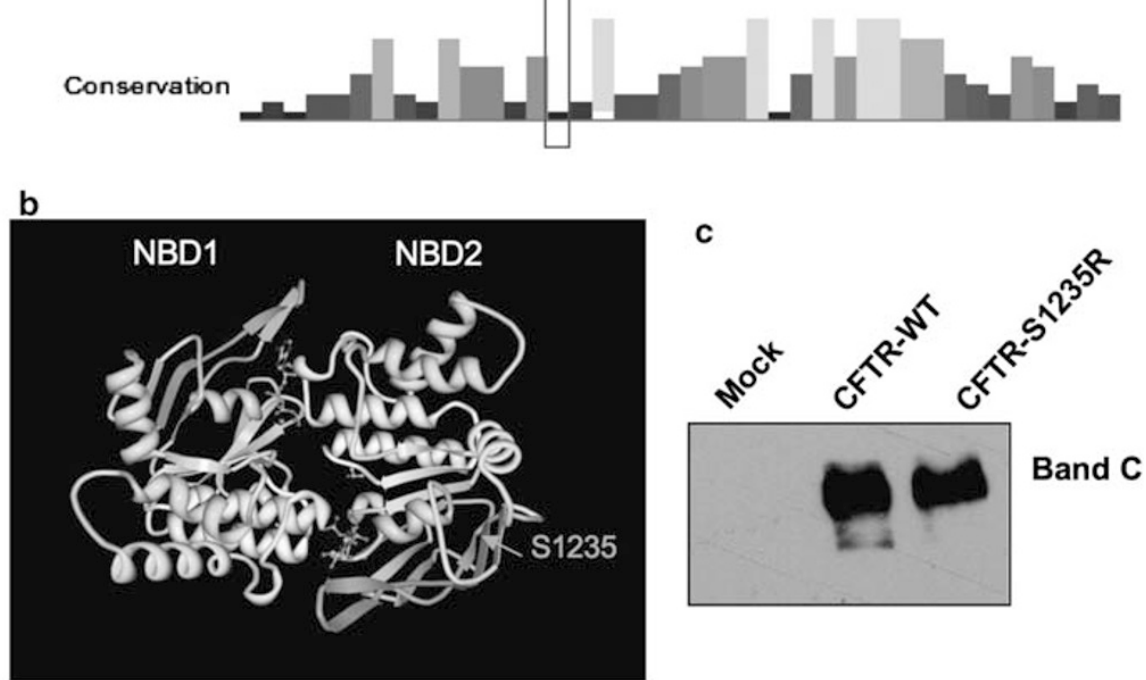

Figure 1 Structural and processing impact of p.Ser1235Arg mutation on the CFTR protein. (a) Conservation of residues close to Ser1235 (blue square) in the two NBD sequences of human CFTR and other ABC transporters. Positions always occupied by hydrophobic amino acids (V, I, L, M, F, Y, W) are shaded blue. Conserved glycine and lysine are colored in orange and in red, respectively. (b) Localization of the serine 1235 on the 3D modelization of the NBD1/NBD2 heterodimer. (c) COS-7 cell lines were transfected with either wild-type or p.Ser1235Arg CFTR constructs. At 48h post-transfection, whole-cell proteins were extracted and subjected to a western blot analysis. Experiments were repeated three times. The color reproduction of this figure is available on the html full text version of the manuscript.

counseling very difficult, especially in the context of prenatal diagnosis. Through genotype/phenotype analysis of patients or individuals carrying p.Ser1235Arg, analyzed by the French network and functional analysis, we present several lines of evidence that argue against an association of p.Ser1235Arg with CF or CBAVD, although a partial penetrance of p.Ser1235Arg could not be ruled out.

First, the presence of a complex allele [p.Ser1235Arg;class I mutation] in trans of a severe mutation in five patients presenting severe disease (Table 1a) strongly suggests that p.Ser1235Arg alone cannot be considered as a CF-causing mutation. Moreover, among the 11 patients referred for CAVD, 9 are compound heterozygous for a mutation and the complex allele [p.Ser1235Arg;(TG)13(T)5]. The (TG)13(T) 5 allele without the contribution of p.Ser1235Arg is sufficient to result in male infertility or in mild phenotypes. ${ }^{24,25,35}$ Among the two patients with p.Ser1235Arg as single allele, one has the mutation p.Phe508del in trans whereas no other mutation was identified in the second one. The possibility of mutations in intronic regions undetectable by our methods cannot be excluded.

Second, we present 14 asymptomatic individuals (Table 1b), of whom 12 carried a severe and 2 a mild mutation in trans. Three, carrying the complex allele [p.Ser1235Arg;(TG)13(T)5] are sisters or brother of CBAVD or CUAVD patients and no respiratory or digestive symptom was reported. In females, allele $(\mathrm{T}) 5$ has been shown to have a low penetrance, ${ }^{36}$ whereas no urogenital data were available for the males because of absence of parental project. Three adult males are compound heterozygous for p.Ser1235Arg and a severe mutation; two (cases 4 and 5) are biological fathers of CF children and transmitted the severe mutation. Cases 7-11 are patients' mothers or relatives with no evidence of CF symptoms. Cases 12 and 13 are non-symptomatic sister and first-cousin of a patient with respiratory symptoms whose CF diagnostic could be discussed because symptoms are close to asthma.

Third, the frequency of the p.Ser1235Arg in the general population (Table 2) is higher than in CF and CBAVD patients, in accordance with previous reports, ${ }^{14,37}$ and is close to the p.Phe508del frequency $(0.82 \%)$. In contrast, in CF or CBAVD groups, the p.Ser1235Arg frequency is significantly lower than p.Phe508del.

From the functional point of view, the results from alignment show that this residue is poorly conserved and occurs in a region not implicated in known CFTR functions. Moreover, in silico predictions suggest that this nucleotide change is not critical for the mRNA splicing and the CFTR function. These data are confirmed by functional studies, which showed that p.Ser1235Arg does not alter the CFTR mRNA correct splicing and the protein maturation. These data are consistent with previous functional study of this locus in combination with alleles found at p.Met470Val and p.Gly628Arg loci. ${ }^{13}$ Wei et al. ${ }^{13}$ demonstrated that the p.Ser1235Arg CFTR protein does not cause change in the chloride transport activity. Besides, the p.Gly628Arg/p.Ser1235Arg mutant protein induces a significantly lower cAMP-dependent chloride transport activity than the 
a
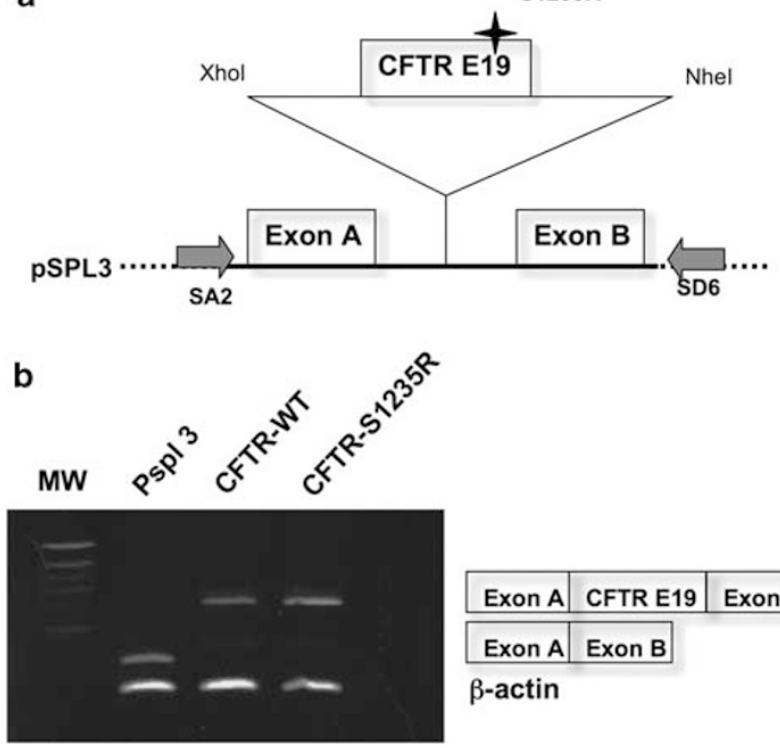

\begin{tabular}{l|l|l|}
\hline Exon A & CFTR E19 & Exon B \\
\hline Exon A & Exon B \\
\hline$\beta$-actin
\end{tabular}

Figure 2 Functional impact on the CFTR mRNA splicing. (a) Schema of the minigene constructs in PSPL3 vector. The exon 19 and exon-intron junctions were inserted in the multiple cloning site of the PSPL3 vector between exons $A$ and $B$ from the $\beta$-globin by PCR restriction. (b) RT-PCR analysis of mRNA of cell expressing wild-type ${ }^{42}$ or p.Ser1235Arg(S1235R) constructs. BeaS2B cells were transfected with either empty, wild-type or p.Ser1235Arg pSPL3 vector. After $48 \mathrm{~h}$, total RNAs were extracted and cDNAs were synthetized. PCR was performed using SA2 and SD6 primers amplifying the region between exons $\mathrm{A}$ and $\mathrm{B}$. On the right, the composition of each transcript was indicated. Each experiment was repeated at least three times.

p.Gly628Arg mutant protein, showing the major importance of genetic background, particularly for missense mutations.

In a second step, we determined the haplotypes linked to p.Ser1235Arg and found the same haplotype in $72.4 \%$ of cases. Only the IVS17b(TA) marker presents a large variability, although three haplotypes, deriving one from the other by addition or deletion of one dinucleotide $(35,36$ or 37 repeats), represent $>55 \%$ of the alleles. Besides, IVS1(CA) 26, a rare allele in the European populations (4.2 vs $80 \%$ for alleles 22,23 and 24), ${ }^{38}$ is strictly linked to p.Ser1235Arg. In CBAVD patients, the haplotype 26-17-13-5-M-33-13 was found in $85.7 \%$ of p.Ser1235Arg alleles. These data suggest the further change of the (TG)12(T)7 to (TG) $13(\mathrm{~T}) 5$ on a chromosome bearing the alteration p.Ser1235Arg on a background IVS17b(TA)33. Together, these data suggest that p.Ser1235Arg could derive from a founding event and haplotypes differing by a single microsatellite could depend on slippage phenomena, as already proposed. ${ }^{39}$

In conclusion, there are now strong lines of evidence against a severe deleterious effect of p.Ser1235Arg and its association with classical CF or CBAVD disease. However, a partial penetrance and/ or the presence of other sequence alterations in non-screened regions particularly in patients with atypical or mild symptoms of $\mathrm{CF}^{27,40,41}$ cannot be ruled out. These data highlight the importance of searching for a complex allele whenever p.Ser1235Arg is identified. The absence of clinical effects in healthy individuals who are compound heterozygous for the p.Ser1235Arg and another severe mutation is helpful to provide adequate genetic counseling to the families with regard to its transmission in offspring.

\section{CONFLICT OF INTEREST}

The authors declare no conflict of interest.

\section{ACKNOWLEDGEMENTS}

We thank Dr C Beroud for fruitful discussion on the software analysis and the GREPAM for efficient anonymized sample referral.

1 Kerem E: Atypical CF and CF related diseases. Paediatr Respir Rev 2006; 7 (Suppl 1): S144-S146.

2 Cotton RG, Scriver CR: Proof of 'disease causing' mutation. Hum Mutat 1998; 12: 1-3. 3 Castellani C, Cuppens H, Macek Jr M et al: Consensus on the use and interpretation of cystic fibrosis mutation analysis in clinical practice. J Cyst Fibros 2008; 7: 179-196.

4 Savov A, Angelicheva D, Balassopoulou A, Jordanova A, Noussia-Arvanitakis S, Kalaydjieva L: Double mutant alleles: are they rare? Hum Mol Genet 1995; 4: 1169-1171.

5 Fanen P, Clain J, Labarthe R et al: Structure-function analysis of a double-mutant cystic fibrosis transmembrane conductance regulator protein occurring in disorders related to cystic fibrosis. FEBS Lett 1999; 452: 371-374.

6 Romey MC, Guittard C, Chazalette JP et al: Complex allele $(-102 T>A+S 549 R(T>G))$ is associated with milder forms of cystic fibrosis than allele $S 549 R(T>G)$ alone. Hum Genet 1999; 105: 145-150.

7 Clain J, Fritsch J, Lehmann-Che J et al: Two mild cystic fibrosis-associated mutations result in severe cystic fibrosis when combined in cis and reveal a residue important for cystic fibrosis transmembrane conductance regulator processing and function. $J$ Biol Chem 2001; 276: 9045-9049.

8 Rohlfs EM, Zhou Z, Sugarman EA et al: The I148T CFTR allele occurs on multiple haplotypes: a complex allele is associated with cystic fibrosis. Genet Med 2002; 4: 319-323.

9 Clain J, Lehmann-Che J, Girodon E et al: A neutral variant involved in a complex CFTR allele contributes to a severe cystic fibrosis phenotype. Hum Genet 2005; 116: 454-460.

10 Niel F, Legendre M, Bienvenu T et al: A new large CFTR rearrangement illustrates the importance of searching for complex alleles. Hum Mutat 2006; 27: 716-717.

11 Claustres M, Altieri JP, Guittard C, Templin C, Chevalier-Porst F, Des Georges M: Are p.I148T, p.R74W and p.D1270N cystic fibrosis causing mutations? BMC Med Genet 2004; 5: 19.

12 Cuppens H, Marynen P, De Boeck C, Cassiman JJ: Detection of $98.5 \%$ of the mutations in 200 Belgian cystic fibrosis alleles by reverse dot-blot and sequencing of the complete coding region and exon/intron junctions of the CFTR gene. Genomics 1993; 18: 693-697.

13 Wei L, Vankeerberghen A, Jaspers M, Cassiman J, Nilius B, Cuppens H: Suppressive interactions between mutations located in the two nucleotide binding domains of CFTR. FEBS Lett 2000; 473: 149-153.

14 Monaghan KG, Feldman GL, Barbarotto GM, Manji S, Desai TK, Snow K: Frequency and clinical significance of the S1235R mutation in the cystic fibrosis transmembrane conductance regulator gene: results from a collaborative study. Am J Med Genet 2000; 95: 361-365.

15 Dequeker E, Stuhrmann M, Morris MA et al: Best practice guidelines for molecular genetic diagnosis of cystic fibrosis and CFTR-related disorders-updated European recommendations. Eur J Hum Genet 2009; 17: 51-65.

16 Oca F, Dreux S, Gerard B et al: Amniotic fluid digestive enzyme analysis is useful for identifying CFTR gene mutations of unclear significance. Clin Chem 2009; 55: 2214-2217.

17 Caggana M, Conroy JM, Pass KA: Rapid, efficient method for multiplex amplification from filter paper. Hum Mutat 1998; 11: 404-409.

18 Cartegni L, Wang J, Zhu Z, Zhang MQ, Krainer AR: ESEfinder: a web resource to identify exonic splicing enhancers. Nucleic Acids Res 2003; 31: 3568-3571.

19 Fairbrother WG, Yeh RF, Sharp PA, Burge CB: Predictive identification of exonic splicing enhancers in human genes. Science 2002; 297: 1007-1013.

20 Sironi M, Menozzi G, Riva L et al: Silencer elements as possible inhibitors of pseudoexon splicing. Nucleic Acids Res 2004; 32: 1783-1791.

21 Wang Z, Rolish ME, Yeo G, Tung V, Mawson M, Burge CB: Systematic identification and analysis of exonic splicing silencers. Cell 2004; 119: 831-845.

22 Eudes R, Lehn P, Ferec C, Mornon JP, Callebaut I: Nucleotide binding domains of human CFTR: a structural classification of critical residues and disease-causing mutations. Cell Mol Life Sci 2005; 62: 2112-2123.

23 Rene $C$, Taulan M, Iral F et al: Binding of serum response factor to cystic fibrosis transmembrane conductance regulator CArG-like elements, as a new potential CFTR transcriptional regulation pathway. Nucleic Acids Res 2005; 33: 5271-5290.

24 Disset A, Michot C, Harris A, Buratti E, Claustres M, Tuffery-Giraud S: A T3 allele in the CFTR gene exacerbates exon 9 skipping in vas deferens and epididymal cell lines and is associated with Congenital Bilateral Absence of Vas Deferens (CBAVD). Hum Mutat 2005; 25: 72-81.

25 Groman JD, Hefferon TW, Casals T et al: Variation in a repeat sequence determines whether a common variant of the cystic fibrosis transmembrane conductance regulator gene is pathogenic or benign. Am J Hum Genet 2004; 74: 176-179.

26 Claustres M: Molecular pathology of the CFTR locus in male infertility. Reprod Biomed Online 2005; 10: 14-41.

27 Feldmann D, Couderc R, Audrezet MP et al: CFTR genotypes in patients with normal or borderline sweat chloride levels. Hum Mutat 2003; 22: 340.

28 Muller F, Simon-Bouy B, Girodon E, Monnier N, Malinge MC, Serre JL: Predicting the risk of cystic fibrosis with abnormal ultrasound signs of fetal bowel: results of a French 
molecular collaborative study based on 641 prospective cases. Am J Med Genet 2002; 110: 109-115.

29 Bombieri C, Benetazzo M, Saccomani A et al: Complete mutational screening of the CFTR gene in 120 patients with pulmonary disease. Hum Genet 1998; 103: 718-722.

30 Casals T, De-Gracia J, Gallego M et al: Bronchiectasis in adult patients: an expression of heterozygosity for CFTR gene mutations? Clin Genet 2004; 65: 490-495.

31 Pallares-Ruiz N, Carles S, Des Georges M et al: Complete mutational screening of the cystic fibrosis transmembrane conductance regulator gene: cystic fibrosis mutations are not involved in healthy men with reduced sperm quality. Hum Reprod 1999; 14: 3035-3040.

32 Claustres M, Guittard C, Bozon D et al: Spectrum of CFTR mutations in cystic fibrosis and in congenital absence of the vas deferens in France. Hum Mutat 2000; 16 : 143-156.

33 Callebaut I, Eudes R, Mornon JP, Lehn P: Nucleotide-binding domains of human cystic fibrosis transmembrane conductance regulator: detailed sequence analysis and threedimensional modeling of the heterodimer. Cell Mol Life Sci 2004; 61: 230-242.

34 Desmet F, Hamroun D, Lalande M, Collod-Béroud G, Claustres M, Béroud C: Human Splicing Finder: an online bioinformatics tool to predict splicing signals. Nucleic Acids Res 2009; 37: e67.

35 Cuppens $\mathrm{H}$, Lin $\mathrm{W}$, Jaspers $\mathrm{M}$ et al: Polyvariant mutant cystic fibrosis transmembrane conductance regulator genes. The polymorphic (Tg)m locus explains the partial penetrance of the T5 polymorphism as a disease mutation. J Clin Invest 1998; 101: 487-496.

36 Sun W, Anderson B, Redman J et al: CFTR 5T variant has a low penetrance in females that is partially attributable to its haplotype. Genet Med 2006; 8: 339-345.

37 Modiano G, Bombieri C, Ciminelli BM et al: A large-scale study of the random variability of a coding sequence: a study on the CFTR gene. Eur J Hum Genet 2005; 13: 184-192.

38 Mateu E, Calafell F, Bonne-Tamir B et al: Allele frequencies in a worldwide survey of a CA repeat in the first intron of the CFTR gene. Hum Hered 1999; 49: 15-20.

39 Claustres M, Desgeorges M, Moine P, Morral N, Estivill X: CFTR haplotypic variability for normal and mutant genes in cystic fibrosis families from southern France. Hum Genet 1996; 98: 336-344.

40 Castellani C, Benetazzo MG, Tamanini A, Begnini A, Mastella G, Pignatti P: Analysis of the entire coding region of the cystic fibrosis transmembrane regulator gene in neonatal hypertrypsinaemia with normal sweat test. J Med Genet 2001; 38: 202-205.

41 Reboul MP, Laharie D, Amouretti M, Lacombe D, Iron A: Isolated idiopathic chronic pancreatitis associated with a compound heterozygosity for two mutations of the CFTR gene. Gastroenterol Clin Biol 2003; 27: 821-824.

42 Robertson NH, Weston SL, Kelly SJ et al: Development and validation of a screening test for 12 common mutations of the cystic fibrosis CFTR gene. Eur Respir J 1998; 12 : $477-482$. 